statistically insignificant. PFOS exposure and birth weight were associated in some studies, while others found no association.

Conclusions The literature suggests no consistent association between in utero exposure to PFOS and birth weight. However, exposure to PFOA is associated with decreased average birth weight in populations with high exposure levels. Considering the global exposure to PFCs, this calls for careful interpretation within a public health perspective.

\section{PO-0353 LICHEN STRIATUS - CASE REPORT}

${ }^{1}$ E Brinzan, ${ }^{2} \mathrm{C}$ Strugaru, ${ }^{3} \mathrm{G}$ Florescu, ${ }^{1} \mathrm{D}$ Popescu, ${ }^{4} \mathrm{~A}$ Kosa, ${ }^{5} \mathrm{CP}$ Popescu, ${ }^{5} \mathrm{~S}$ Florescu, ${ }^{5} \mathrm{EM}$ Ceausu, ${ }^{5} \mathrm{P}$ Calistru. ${ }^{1}$ Pediatrics, Dr v Babes Foundation, Bucharest, Romania; ${ }^{2}$ Genetics, Carol Davila University of Medicine and Pharmacy, Bucharest, Romania; ${ }^{3}$ Dermatology, Dr v Babes Foundation, Bucharest, Romania; ${ }^{4}$ Cinical Research, Dr v Babes Foundation, Bucharest, Romania; ${ }^{5}$ Infectious Diseases, Carol Davila University of Medicine and Pharmacy, Bucharest, Romania

10.1136/archdischild-2014-307384.1001

Background Lichen striatus is an common dermatosis that follows the lines of Blaschko. Aetiology of this eruption is unknown, its features are less known to paediatricians.

Objective We attempted to characterise the clinical, pathologic features and evolution of one case presented to the paediatric departament in our clinic.

Materials and methods A 2-year-old girl, atopic, presented with fever and upper respiratory tract infection.

Clinical examination: one month long dull-red papulo-squamous dermatosis arranged in a Blaschko line. The eruption appeared suddenly a month ago. The skin lesions were asymmetrically distributed linearly unilateral along the left arm, arranged in a Blaschko line pattern. Intense itching was present in this case.

Laboratory results: $\mathrm{Hb}=12,6 \mathrm{~g} / \mathrm{dl}, \mathrm{Ht}=35,2 \%$, $\mathrm{WBC}=9400 / \mu \mathrm{L}, \mathrm{S}=25 \%$, Eo $=2,5 \%, \mathrm{Ba}=0,745 \%$, $\mathrm{Ly}=71,1 \%, \mathrm{RBC}=4,38 \times 10 \mathrm{mil} / \mu \mathrm{L}, \mathrm{PLT}=336000 \mu \mathrm{L}$, $\mathrm{ALT}=23 \mathrm{U} / \mathrm{L}, \mathrm{AST}=31 \mathrm{U} / \mathrm{L}, \mathrm{CRP}=0,6 \mathrm{mg} / \mathrm{dL}$; Creatinin, Immunogram, Glucose -normal; HIV $1+2$, Ag HBs, Anti $\mathrm{HCV}$-negative

Histopathology revealed features consistent with lichen striatus.

Tratament for itchy lichen striatus consisted in topical steroid application (mometasone furoate 1\% cream once daily for two weeks).

Five months later, complete resolution of the skin lesions occurred without any other treatment, leaving only slightly hypopigmented macules on the left arm.

Conclusion

1. That lesions of lichen striatus may have a prolonged active phase, the differential diagnosis includes other inflammatory diseases that can assume a linear pattern, paediatrician and dermatologist must team up in these cases;

2. Post-inflammatory hypopigmentation can be a cause for concern for the parents and their children.

\section{PO-0354 CHRONIC BULLOUS DISEASE OF CHILDHOOD (CBDC) OR LINEAR IGA DERMATOSIS}

${ }^{1} Y$ Castillo Avila, ${ }^{2}$ Ml Martinez de Pablo, ${ }^{1}$ A Guell Bara, ${ }^{1}$ A Diaz Conradi. ${ }^{1}$ Pediatria, Fundacion Hospital de Nens de Barcelona, Barcelona, Spain; ${ }^{2}$ Dermatologia Pediatrica, Fundacion Hospital de Nens de Barcelona, Barcelona, Spain
Introduction Linear IgA dermatosis is an autoimmune bullous disease characterised by the presence of linear deposits of IgA in the dermoepidermal junction. Predominates in the first decade of life. It produces blisters that tend to be distributed in rings or cluster of jewels.

Case Report 4 year old boy with generalised bullous lesions on face, trunk and limbs, which are grouped in rings. No palmoplantar involvement or mucous membranes with good condition. 10 days before diagnosed of chickenpox. He had also been treated with amoxicillin- clavulanic acid for otitis media.

On suspicion of bullous impetigo he was hospitalised. Paediatric dermatology oriented probable diagnosis, bullous disease linear IgA dermatosis type vs bullous pemphigoid and practiced skin biopsy. Initial treatment oral prednisone at doses of $1 \mathrm{mg} / \mathrm{kg}$ and topical mupirocin.

Results Direct immunofluorescence confirmed the linear IgA deposits at the dermoepidermal junction. Sulfone $(1,3 \mathrm{mg} / \mathrm{kg})$ was added to oral corticosteroid treatment, in order to remove corticosteroids at long-term. Three months later the child is in remission from his lessions. The evolution will determine whether it can be considered a case of primary or secondary CBDC, primary cases tend to recur in months or years

Conclusions We present a case of a child with typical lesions of CBDC, with good response to steroids and sulfone.

At the time of diagnosis he was treated with amoxicillin- clavulanic because of infectious process. There are reported cases of CBDC secondary to viral, bacterial processes and drugs, such like amoxicillin and clavulanic.

\section{PO-0355 HENOCH-SCHÖNLEIN PURPURA: CLINICAL COURSE TO 6 MONTHS OF FOLLOW}

C Castillo Rukoz, S Pons Morales, C Salido Capilla, M Salamanca Campos, J Fujkova, M Marti Masanet. Paediatric, Hospital Universitario Dr Peset, Valencia, Spain

\subsection{6/archdischild-2014-307384.1003}

Background and aims The Henoch-Schönlein Purpura (HSP) is the most common of small-vessel vasculitis in children, with an annual incidence of 20.4/100. 000 in $<17$ years. The pathogenesis is unknown, but exposure to various antigens (infectious agents, vaccines, drugs) is considered as a possible immune factor trigger.

Methods Retrospective descriptive study by review of medical records of patients diagnosed with HSP between 2002-2013, and followed in rheumatology consultations for at least 6 months.

Results 119 children with a mean age at diagnosis of 6.1 years (range 1-14 years) were controlled.

Symptoms, blood pressure and urine dipstick were recorded at baseline and monthly during six months.

A previous infectious process was observed in 43 cases (36\%): 10 streptococcus, 1 EBV, 2 Mycoplasma, 2 adenovirus and one parvovirus.

Purple was the first clinical sign in $90 \%$. Of extrarenal symptoms, arthralgia appeared in $40(33.6 \%)$ patients during followup, affecting more frequently ankles and knees (84\%). The scrotal oedema occurred in 4 children, 2 had oedema of scalp and one on the sacrum. The abdominal pain was present in 18 cases (15\%), 6 with ultrasound suggestive of intestinal wall vasculitis. The isolated microscopic hematuria was seen in 18 cases (15\%), presenting normal blood pressure, 3 of these patients developed nephropathy in the next 2 months. 
After 6 months of evolution all symptoms were resolved, with persisting hematuria in cases of nephropathy.

Conclusions HSP is usually a self-limiting disease. The extrarenal symptoms usually resolve quickly, without complications, and long-term prognosis depends mainly on the severity of renal involvement.

\section{PO-0356 HOW FREQUENT GET TUBERCULOSIS A CF PATIENT}

I Ciuca, L Pop. Pediatric II Department, "Victor Babes" University of Medicine and Pharmacy, Timisoara, Romania

\subsection{6/archdischild-2014-307384.1004}

Background Cystic fibrosis patients are predisposed to pulmonary infections. Conditions associated with $\mathrm{CF}$, like underweight, diabetes mellitus (CFRD), liver disease (CFLD) are favouring factors for tuberculosis (TB). The hypothesis of a potential comorbidity of TB in CF children occurred. The aim of the paper was to evaluate the prevalence of $\mathrm{TB}$ in children with complicated CF.

Methods Thirty-two patients (pts) with typical CF, associating complication like CFLD(27 pts), diabetes (3 pts) and 2 with both complication were considered for a prospective five years study. Biannual bacteriologic exam (TB specific also) were included, in addition to clinical examination and annual CT, to the patients evaluation.

Results Tuberculosis occurred in 6.25\% (2 patients), both F508 del homozygous, with CFLD and poor nutritional status; one patient had also CFRD. His evolution was unfavourable; he developed portal hypertension and died from respiratory failure. The other patient was diagnosed with active TB, Pseudomonas positive and poor nutritional status, but good evolution after treatment. The rest of CFLD patients had a stationary evolution, except 4 of them $(15.38 \%)$ developed diabetes. Tuberculin skin test was positive in 4 patients (12.5\%), 3 of them received TB vaccine. Despite the mandatory vaccination for TB in our country, only $84 \%$ pts were vaccinated. $18.75 \%$ of patients (6 pts) were considered and treated as TB cases, without bacteriological confirmation, before being diagnosed with CF.

Conclusion Although TB is a frequent condition in our area, the prevalence among CF children is not as high as expected. It is possible that other factors, unknown so far, are implicated.

\section{P0-0357 PRENATAL EXPOSURE TO ENDOCRINE DISRUPTING CHEMICALS (EDCS) IN RELATION TO THYROID HORMONE LEVELS IN INFANTS}

${ }^{1} \mathrm{M}$ de Cock, ${ }^{2} \mathrm{MR}$ de Boer, ${ }^{3} \mathrm{M}$ Lamoree, ${ }^{3} \mathrm{~J}$ Legler, ${ }^{1} \mathrm{M}$ van de Bor. ${ }^{1}$ Health and Life Sciences, VU University, Amsterdam, Netherlands; 'Health Sciences, VU University, Amsterdam, Netherlands; ${ }^{3}$ Institute for Environmental Studies, VU University, Amsterdam, Netherlands

\subsection{6/archdischild-2014-307384.1005}

Background The presence of chemicals in the environment is ubiquitous. EDCs in particular may disrupt thyroid hormones, which in early life are essential for brain development. As observational studies regarding this topic are still limited, the objective of the current study was to assess the association between prenatal exposure to various EDCs and thyroxine (T4) levels in newborns in a mother-child cohort in the Netherlands.
Methods Exposure to amongst others dichlorodiphenyldichloroethylene (DDE), perfluorooctanesulfonic acid (PFOS), and perfluorooctanoic acid (PFOA) was determined in cord plasma or breast milk, and information on T4 levels in heel prick blood spots was obtained through the Dutch neonatal screening programme. Linear regression models were composed for each compound and T4. Models were stratified for gender and adjusted for a priori defined covariates.

Results Mean T4 level was $86.9 \mathrm{nmol} / \mathrm{L}(\mathrm{n}=83)$. Girls in Q4 of DDE and PFOA exposure showed an increased T4 level compared to Q1, a difference which remained significant in the fully adjusted model (DDE $\geq 110.01 \mathrm{ng} / \mathrm{L},+22.2 \mathrm{nmol} / \mathrm{L}, 95 \% \mathrm{CI}$ $1.37,43.04 ;$ PFOA $\geq 1201 \mathrm{ng} / \mathrm{L},+30.7 \mathrm{nmol} / \mathrm{L}, 95 \%$ CI 13.38 , 48.05). In boys a lower T4 level was seen in Q2 for both PFOS and PFOA, however after adjusting the models these associations were attenuated.

Conclusion DDE, PFOS and PFOA were associated with T4 in a sex-specific manner. As the study population was relatively small, results should be considered as exploratory. More research is warranted, as studies on the role of exposure to environmental contaminants in thyroid hormone homeostasis are still limited.

\section{PO-0358 CONCOMITANT CEFTRIAXONE-INDUCED NEPHROLITHIASIS AND BILIARY PSEUDOLITHIASIS IN A SMALL TODDLER}

${ }^{1} \mathrm{~S}$ Diaconescu, ${ }^{1} \mathrm{~A}$ Baltag, ${ }^{2} \mathrm{C}$ Olaru, ${ }^{1} \mathrm{~N}$ Gimiga, ${ }^{1} \mathrm{M}$ Burlea, ${ }^{1} \mathrm{CA}$ Olaru, $1 \mathrm{I}$ Miron. "Pediatrics, "Gr. T. Popa" University of Medicine and Pharmacy, Jassy, Romania; ${ }^{2}$ Pediatrics, "St. Mary" Children's Emergency Hospital, Jassy, Romania

\subsection{6/archdischild-2014-307384.1006}

Introduction Ceftriaxone is a frequent choice in Romanian hospitals for empiric antimicrobial therapy because of its broad spectrum, long half-life, safety and tolerability. However, some relatively rare side effects as renal lithiasis and biliary pseudolithiasis may occur.

Case report A 5 month male toddler was admitted into our unit with extreme agitation and mild respiratory symptoms. The child had interstitial pneumonia and received ceftriaxone intravenously for 7 days in a county hospital. Clinical examination showed productive cough, fine crackles, mild systolic murmur and small crystals in the in diaper. Ultrasound examination of the kidneys revealed the presence of sediment in the bladder and a hyperechoic image of $4 \mathrm{~mm}$ in the gallbladder. Complete blood count, urea, creatinine, creatinine clearance, serum glucose, calcium (total and ionised), serum phosphorus, bilirubinemia, alkaline phosphatase were within normal limits. Urinalysis showed calcium carbonate deposit and increased calcium/creatinine ratio. Chest X-Ray revealed discrete bilateral interstitial infiltrate. The echocardiography found permeable foramen ovale and moderate left ventricular diastolic dysfunction. Treatment consist in suspending ceftriaxone administration, antispasmodics, hydrochlorothiazide $6,25 \mathrm{mg} /$ daily and adequate hydration. The child was dismissed after 3 days in good general condition, disappearance of bladder sediment but persistant gallbladder image that disappeared within 3 weeks.

Conclusions This is a particular case of a very young patient with renal and vesicular pseudolithiasis induced by ceftriaxone; the negative family history of kidney stones, a normal intake of vitamin D3 and normal liver and renal function excluded any other cause of lithiasis except the antibiotic itself. 